TECHNICAL NOTE

\author{
T.P. Duprez \\ A. Jankovski \\ C. Grandin \\ L. Hermoye \\ G. Cosnard \\ C. Raftopoulos
}

\section{Intraoperative 3T MR Imaging for Spinal Cord Tumor Resection: Feasibility, Timing, and Image Quality Using a "Twin" MR-Operating Room Suite}

SUMMARY: We assessed feasibility, safety, and timing of an original intraoperative MR procedure in 3 cases of resection of spinal cord glioma by using a clinical 3T MR system connected to an adjacent operating room in a design being coined "twin" or "dual" MR-operating room suite.
C erebral intraoperative MR imaging (IoMRI) is nowadays gaining wide acceptance and larger availability. ${ }^{1,2}$ Different designs of operating theaters and MR systems have been proposed for IoMRI in the past decade. ${ }^{3-7}$ The usefulness of cerebral IoMRI has been assessed in large patient series. ${ }^{8-10} \mathrm{Up}$ to now, spinal cord IoMRI has not been reported yet.

\section{Technique}

\section{Materials and Procedure}

An original complex was built in our institution connecting a clinical 3T MR system (Achieva 3T, Release 2.3.1; Philips Health Care, Best, the Netherlands) to a neurosurgical operating room (OR) through an intermediate lock chamber. Patient transportation back and forth between OR and MR table was enabled by a fully motorized VIWAS operating table (Vascular Interventional Workplace for Advanced Surgery, Maquet, Rastatt, Germany) abutting the doors of the lock chamber opening onto the MR room. Connecting home-manufactured table guides put on both MR and operating tables allowed fast and safe transfer of the patients in operative conditions. Each time an IoMRI procedure was requested, the clinical activity of the MR system was interrupted. After surgical cleaning of the MR room, a fully automated security procedure was activated for switching the MR room from diagnostic to surgical technique. After having performed more than 50 relevant intraoperative examinations for brain tumor resection, we tried a similar procedure for spinal cord gliomas by using paired midsized flexible surface coils of $14 \mathrm{~cm}$ in diameter (SENSE Flex M coil; Philips Health Care). Patient transfers were performed by a zero-drilled team, including 1 anesthesiologist, 1 neurosurgeon, and 2 nurses. The OR table was placed in horizontal full-flat position. Paired flexible surface coils wrapped into sterile drapes were positioned with closest adjustment to the patient's skin by the surgeon, within the OR and before the transfer. The patient, whose head was fixed in an MR-compatible Mayfield head holder (Radionics, Burlington, Mass), was thereafter disconnected from a respirator and

Received December 10, 2007; accepted after revision January 21, 2008.

From the Departments of Radiology and Medical Imaging (T.P.D., C.G., G.C.) and Neurosurgery (A.J., C.R.) and Laboratory of Image Processing (L.H.), Université Catholique de Louvain, Cliniques Universitaires Saint-Luc, Brussels, Belgium.

Please address correspondence to Thierry P. Duprez, Radiology and Medical Imaging, Université Catholique de Louvain, Cliniques Universitaires Saint-Luc, Ave Hippocrate 10, 1200 Brussels, Belgium; e-mail: Thierry.Duprez@uclouvain.be

DOI 10.3174/ajnr.A1134 manually ventilated with inhalational anesthesia during transfer from the OR to MR table. Uninterrupted monitoring of vital parameters during transfer was performed using an MR-compatible anesthesia-monitoring system (Veris; Medrad, Indianola, Pa). The original OR tabletop had been duplicated before the intervention by an additional MR-compatible one made of polyoxymethylene and manufactured by the center for research in mechatronics of our institution, which was able to glide on a prototypic "receiving" MR tabletop designed and manufactured by the same center. When brought onto the MR tabletop, the patient was reconnected to the MR-compatible Veris system.

\section{Safety Issues}

All of the persons involved in patient transfers (surgeons, anesthesiologists, nurses, technicians, etc.) had received appropriate training at preventing any intrusion of a ferromagnetic object within the $0.5-\mathrm{mT}$ field line of the magnet. All of the ferromagnetic objects were removed from the operative field before transfer to the MR table. The coils were drilled to prevent any loop in coils wires and to avoid any contact between coils and the patient's skin. Regarding aseptia, a fast but surgical cleaning of the MR room lasting 10 minutes was performed immediately before the initiation of the each IoMRI procedure by using an antiseptic water solution (Incidin; Ecolab, Brussels, Belgium). The MR room was equipped with easy-to-clean surfaces and a pulsation ceiling system allowing laminar airflow distribution. Airflow was managed electronically with slightly higher air pressure within the OR during clinical activity and slightly higher pressure within the MR room during IoMRI. "Surgical" air quality was obtained by 25-times-per-hour air renewal and terminal filtration. The VIWAS OR table moved on rail tracks and was mechanically buffered to protect the patient's head from vibrations. Access to the MR-OR suite was restricted during the IoMRI procedure by an electronic managing system coupled to that of the airflow, which locked the outside doors of the MR-OR suite. The doors of the intermediate lock area could be opened only after completion of the airflow reversal procedure (from higher pressure in the OR to higher pressure in the MR room), which took almost 20 minutes.

\section{Illustrative Case Notes}

Case 1. The patient was a 26-year-old man presenting with a spinal cord glioma extending from C5/C6 to T7 (Fig 1A). The only goal in this initial patient was to assess the feasibility of spinal IoMRI by obtaining at least one relevant intraoperative image and to determine potentially limiting factors of the procedure. For security reasons, the 

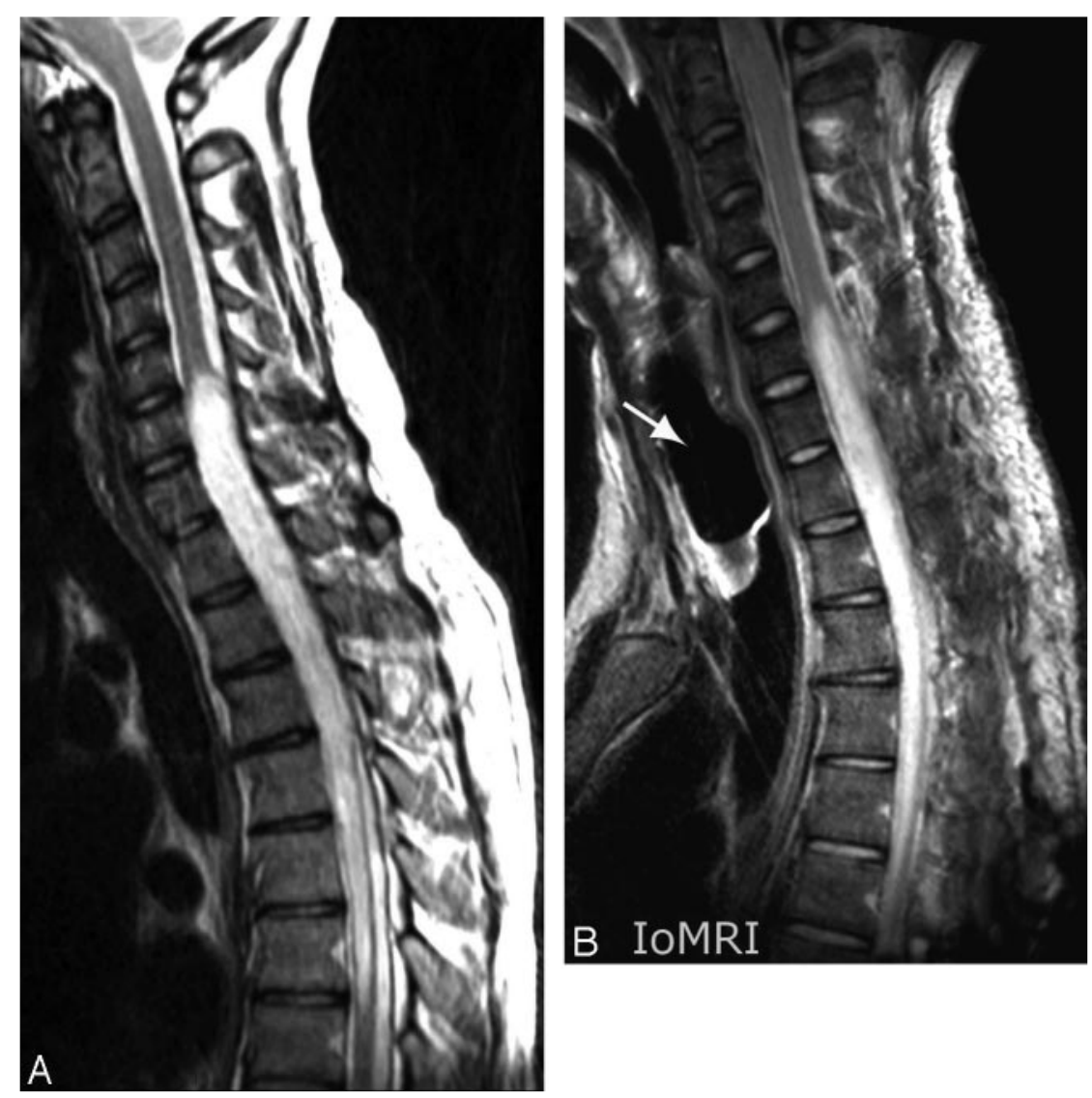

examination was performed after complete closing of the surgical wound. Similar to the position during surgery, the patient was lying in a prone position during IoMRI, with foam padding and blankets to protect bone eminences from overpressure and to favor abdomen breathing, thereby reducing spinal cord movements during surgery and imaging. The whole procedure (from the end of skin closure to reinstallation in the OR) lasted 55 minutes. Most of the time was spent trying many coil positions to enable the sensitivity-encoding (SENSE) parallel imaging (PI) option. However, the OR team failed to obtain sufficient overlapping between elements, and the coils were ultimately placed close together on the midline of the patient's back at the level of the surgical wound. Aside from unfruitful attempts at optimizing coil positioning due to inexperience, no significant limiting factor was encountered. A high-quality sagittal fast spin-echo (FSE) T2-weighted without PI option was finally obtained (Fig 1B).

Case 2. A 44-year-old woman with an ependymoma at the T1-T2 level was the second patient to undergo the procedure. Preoperative work-up was performed by using the same $3 \mathrm{~T}$ system and the same coils as for IoMRI. One element was put on the chest wall and the other on the upper part of the patient's back, just as was planned for IoMRI, to allow a comparison between preoperative and intraoperative image quality (IQ) by using similar pulse sequence parameters (PSPs). Opposite positioning of the 2 coils allowed a SENSE option. A SENSE acceleration factor of 1.4 was set for sagittal acquisitions and of 1.6 for axial transverse ones. A cystic spinal cord ependymoma was present at the T1 and T2 levels with parenchymal edema extending upward and downward (Fig 2A,-B). Similar to the first patient, she had IoMRI after complete closure of the operative field. The patient was turned down from the prone to supine position before IoMRI to reproduce preoperative positioning. Additional steps in the IoMRI procedure were performing precontrast and postcontrast FSE T1-
Fig 1. Case 1. A, Preoperative midsagittal FSE T2-weighted image at 1.5T (TR, $4000 \mathrm{~ms} ; \mathrm{TE}, 75.8 \mathrm{~ms}$; echo train length (ETL) 20; 2 NEX; acquisition time (AT), 3'36" for 11 4-mmthick sections): swelling and abnormal hyperintensity of the diseased segment of the cord were obvious. $B$, Intraoperative midsagittal FSE T2-weighted image at $3 \mathrm{~T}$ after closure of the surgical wound. The 2 elements had been put on the midline of the patient's back in the cranial-caudal direction without capability of SENSE acquisition because of insufficient overlapping between coils. Inflatable balloon of the laryngeal tube was visible (arrow). I0 was rated excellent by using following PSPs: TR, 2209 ms; TE, 120 ms; ETL 20, 4 NEX; no SENSE acceleration factor; AT: 4'56" for 11 4-mm-thick sections.

and T2-weighted images in sagittal and transverse planes. The whole procedure lasted 60 minutes. The IQ of intraoperative images was overall excellent and rated comparable with that of the preoperative images, though signalto-noise ratio (SNR) and contrast-to-noise ratio (CNR) calculations showed slightly lower performances of intraoperative imaging (compare Figs $2 A$ and $2 C$, and Figs $2 B$ and $2 D$ ). Enhanced tumor residue was not detected, but a significant pitfall was highlighted: air bubbles trapped within the spinal canal at the upper extremity of the resection site mimicked the hemosiderin-containing "epidural cap" because of susceptibility artifacts (arrow on Fig $2 C,-D)$.

Case 3. A 48-year-old man had an IoMR procedure during resection of an ependymoma extending from $\mathrm{C} 0$ to T7 (Fig $3 A,-B$ ). Similar to the first patient, he was imaged in a "surgical" prone position, with his head fixed in the Mayfield head holder. The 2 coils were placed in opposition, just as for the previous patient. This was the first attempt at performing a "true intraoperative" spinal cord MR imaging with an unclosed surgical field. A Gelfoam roll (Pfizer, New York, NY) was plugged on the open dura, and only a few loose points were made at the skin to stabilize the Gelfoam plug during the procedure. High-quality precontrast and postcontrast FSE T1and FSE T2-weighted IoMR images were obtained in the sagittal plane (Fig 3C-E). Serial precontrast and postcontrast (2 time points) FSE T1-weighted images in the transverse plane were also obtained (Fig $3 F-H)$. An early enhancement at the margins of the resection cavity was observed that dramatically increased with time leading to an almost complete filling of the cavity by the contrast agent 42 minutes after intravenous perfusion (delay of the last coronal postcontrast T1-weighted acquisition-ending imaging session; not illustrated). A diffusion tensor imaging sequence was acquired in an axial transverse plane with coherent color-coded information regarding the cranialcaudal orientation of the major white matter tracts of the cord and regarding the central color defect corresponding with the resection cavity (not illustrated). This comprehensive and true IoMRI procedure lasted 105 minutes.

\section{Discussion}

The concept of IoMRI was pioneered in 1996 by Black et $\mathrm{al}^{11}$ at the Brigham and Women's Hospital in Boston. The initial prototypic design was that of a dedicated and specially designed "double-doughnut" 0.5T MR system fully included into the OR and performing only cerebral intraoperative examinations. ${ }^{1}$ Several research teams using systems running at 

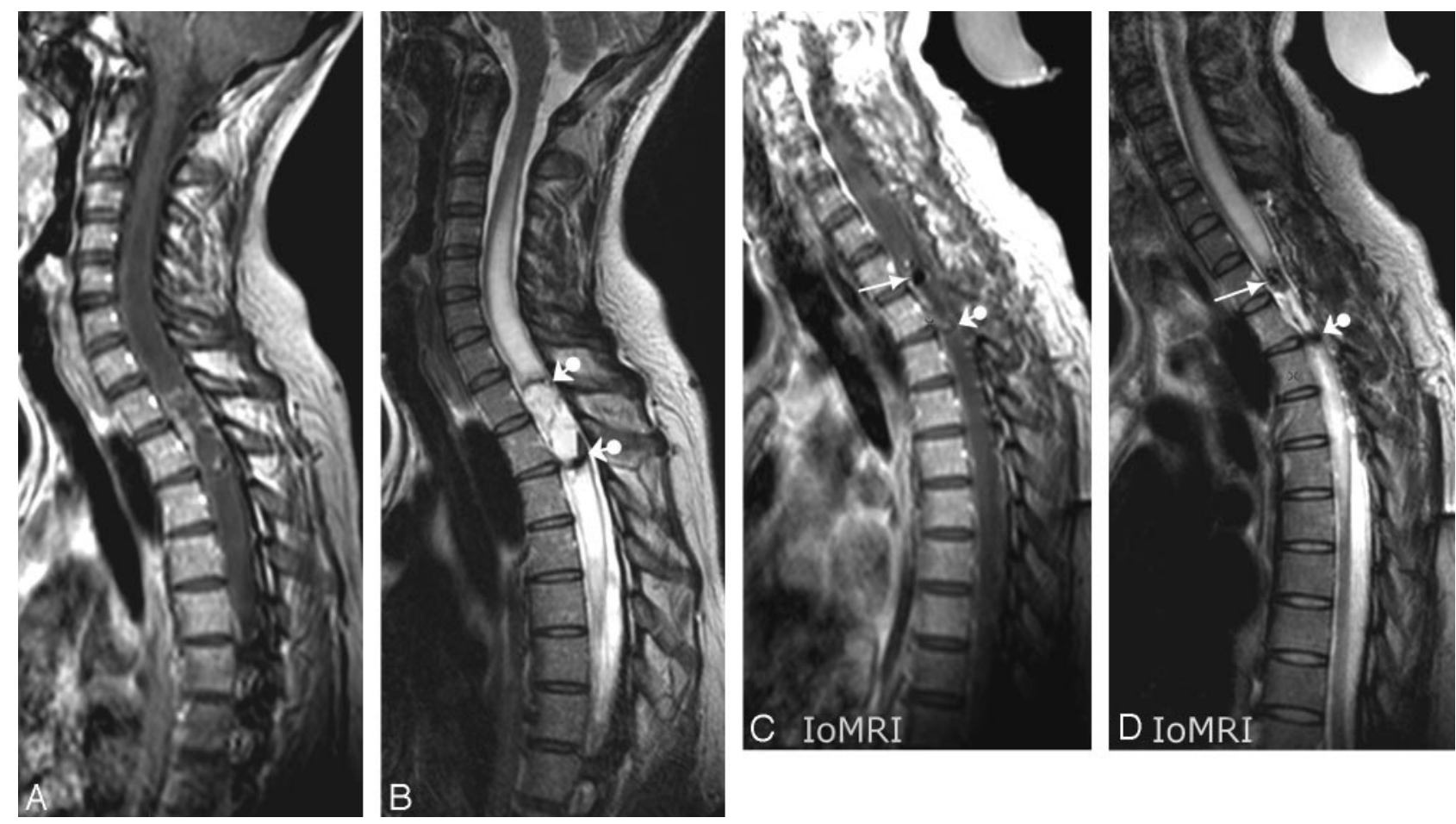

Fig 2. Case 2. Preoperative images. Midsagittal contrast-enhanced FSE T1- $(A)$ and T2-weighted (B) views showed the usual features of spinal cord ependymoma at the T1-T2 level exhibiting pathognomonic hemosiderin-containing epidural "caps" (ball arrowheads in B). PSPs for T1-weighted sequence were as follows: TR, $530 \mathrm{ms;}$;E, $7.8 \mathrm{ms;}$; ETL, 4; 4 NEX; SENSE acceleration factor, 1.4; AT, 4'04" for 11 4-mm-thick sections. PSPs for T2-weighted sequence were as follows: TR, 2209 ms; TE, 120 ms; ETL, 20 ms; 4 NEX; SENSE acceleration factor, 1.4; AT: 3'28" for 11 4-mm-thick sections. On the T2-weighted image (B), SNR for cord and CSF were 119 and 301, respectively. Cord/CSF CNR was 182. C, Intraoperative midsagittal contrast-enhanced FSE T1-weighted view (similar section location and PSP as A) showed trapped air bubble at upper pole of resection site (arrow) and unmodified appearance of inferior hemosiderin-containing epidural cap (ball arrowhead). D, Intraoperative midsagittal FSE T2-weighted view (similar section location and PSP as B) again showed air bubble mimicking epidural (arrow) and unchanged true inferior epidural cap (ball arrowhead). SNRs for cord and CSF were 58 and 194, respectively. Cord/CSF CNR was 135.

basic magnetic fields (B0) ranging from $0.2 \mathrm{~T}$ to $3 \mathrm{~T}$ have investigated various techniques and architectural designs for IoMRI. ${ }^{3-7}$ Significant data assessing the value of the procedure in terms of completeness of brain tumor resection have been published in the past decade. ${ }^{8-10}$ At present, the most common design in use is that of an independent but standard MR system fully included in a magnet-safe OR. In a few institutions, like the hospitals of the Minneapolis-Saint Paul, Minn area, the field strength of the $\mathrm{B} 0$ is now reaching $3 \mathrm{~T}^{12}$

Recently, a twin or dual MR-OR suite allowing independent activity of a nonmagnet-safe OR and of a clinical 3T MR system was built in our institution with the capability of fast and secure switching from a clinical to intraoperative technique. A similar design has just been implemented at the Mayo Clinic (Rochester, Minn). ${ }^{13}$ After more than 50 successful cerebral IoMRIs, we tested the procedure in 3 patients undergoing resection of a spinal cord glioma by using the same surface coils as for cerebral IoMRI. Clinically relevant information could already be drawn from this preliminary experience. First, feasibility and safety of spinal cord IoMRI by using a dual MR-OR suite and standard flexible surface coils without the need for additional spine-dedicated hardware equipment were demonstrated. The second piece of information was that acceptable times for securely transferring the patient back and forth from the OR to the $\mathrm{MR}$ room were required. With more training, we believe that 15-20 minutes would be needed from interrupting surgery to initiating image acquisition and 5-7 minutes from imaging termination to full reinstallation of the patient within the OR. If the 20-minute procedure for airflow reversal is started early with a maximal overlapping with the pa- tient's preparation to transfer, the full time penalty (lost for surgery and without imaging) would have been restrained to approximately 30 minutes. The third piece of information was that high-quality intraoperative spinal images were available at $3 \mathrm{~T}$ in all 3 of the cases by using paired flexible surface coils and standard FSE PSPs as for routine spinal imaging. IQ was preserved even when the PI option was not allowed because of insufficient overlapping between the 2 elements because of the high SNR offered by $3 \mathrm{~T}$ (case 1). When allowed by opposite positioning of the coils, a SENSE acceleration factor of 1.4 for sagittal images and of 1.6 for transverse images was satisfactory regarding IQ (cases 2 and 3). The fourth piece of information was that air trapped within the surgical field could mimic hemosiderin-containing epidural caps of ependymoma, thereby potentially leading to inexact assessment of the true limits of the tumor. In addition, one may speculate the differential diagnosis between susceptibility artifacts due to air bubbles and artifacts due to freshly extravased deoxyhemoglobin-containing blood might be difficult. The fifth piece of information was that contrast enhancement within margins of resection cavity was present at early phases of intraoperative imaging, thereby impeding the accurate delineation of a true tumor residue. In addition, the cavity was demonstrated to be almost completely filled by contrast agent transudation at approximately 42 minutes after perfusion. This phenomenon has already been reported in brain tumor resection. ${ }^{14}$ This could be the major limitation of the clinical value of IoMRI in spinal cord neoplasms, but further experience is warranted. In conclusion, this initial experience in 3 patients demonstrated feasibility and high IQ of spinal cord IoMRI at 3T for tumor resection by using 

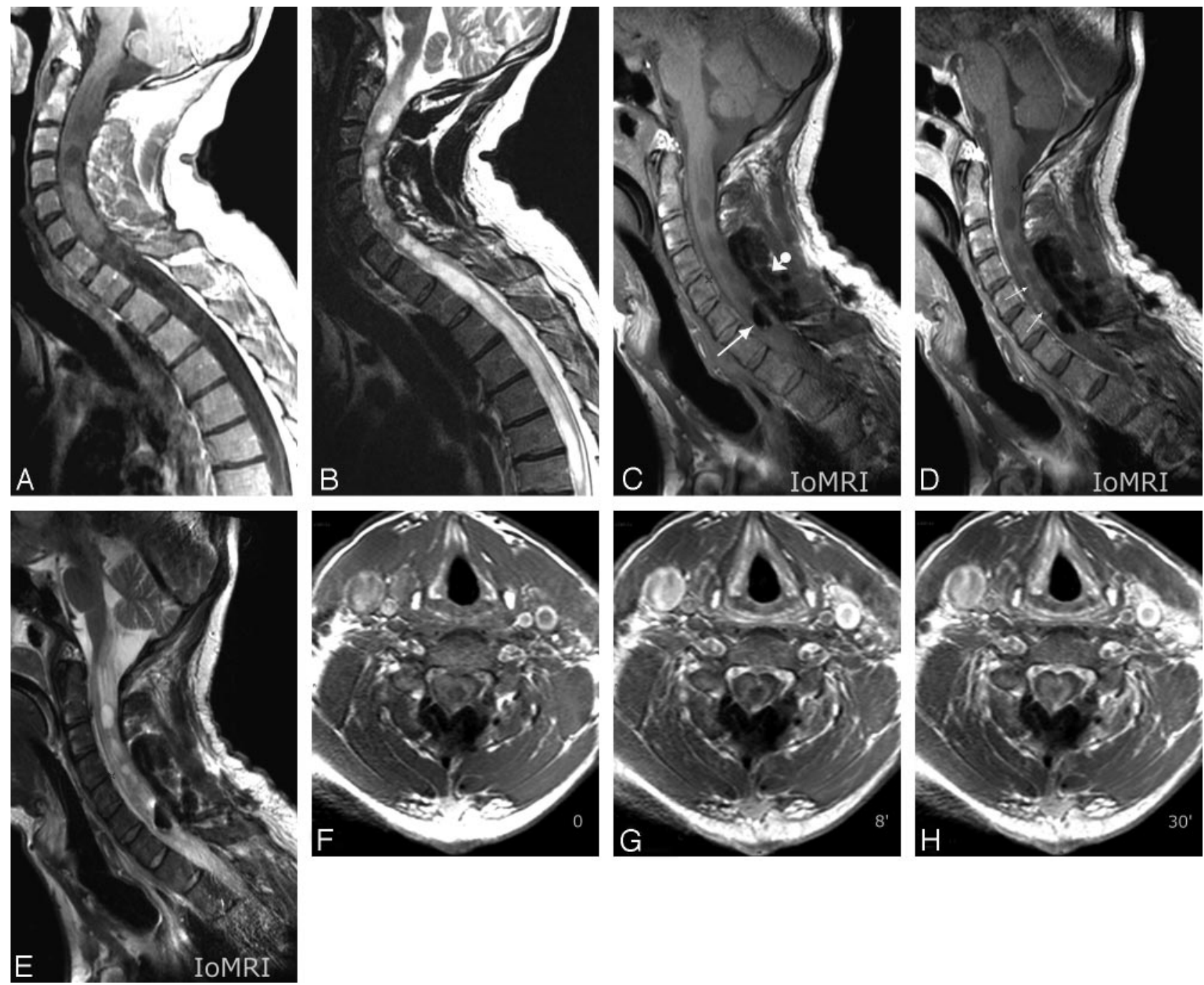

Fig 3. Case 3. Preoperative images. Midsagittal postcontrast FSE T1- $(A)$ and T2-weighted (B) views showing usual features of a spinal cord glioma. C-E. Intraoperative images. Midsagittal precontrast FSE T1-weighted (C), postcontrast FSE T1-weighted (D), and FSE T2-weighted views: observe similar phenomenon of air bubble trapping within surgical field (arrow in C) as in case 2 and contrast-enhancement of the margins of the resection cavity (thin arrows in $D$ ) at this early postcontrast phase of the intraoperative MR procedure. Observe true intraoperative conditions with a Gelfoam roll plugged on open dura (ball arrowhead in C. Similar PSPs as for the previous patient were used. F-H, Intraoperative images. Serial transverse FSE T1-weighted sections at the level of $\mathrm{C} 7$, before contrast agent perfusion $(F)$, at 8 minutes $(G)$, and at 30 minutes $(H)$ after perfusion: striking increase in thickness of enhanced margins of the resection cavity were obvious comparing $F$ to $G$ and H. PSPs were as follows: TR, $530 \mathrm{~ms}$; TE, $7.8 \mathrm{~ms}$; ETL, 4; 4 NEX; SENSE acceleration factor, 1.6; AT, 3'15" for 16 4-mm-thick sections.

paired flexible surface coils but also suggested significant limitations of the technique, mainly due to air bubbles trapping within the operating field and rapidly increasing contrast enhancement of the margins of the resection cavity, together with fast leakage of the contrast agent into the fluid filling the cavity.

\section{References}

1. Jolesz FA. Future perspectives for intraoperative MRI. Review. Neurosurg Clin N Am 2005;16:201-13

2. Hall WA, Truwit CL. Intraoperative MR imaging. Review. Magn Reson Imaging Clin N Am 2005; 13:533-43

3. Schenck JF, Jolesz FA, Roemer PB et al. Superconducting open-configuration MR imaging system for image-guided therapy. Radiology 1995;195:805-14

4. Hadani M, Spiegelman R, Feldman Z, et al. Novel, compact, intraoperative magnetic resonance imaging-guided system for conventional neurosurgical operating rooms. Neurosurgery 2001;48:799-807

5. Schulder M, Liang D, Carmel PW. Cranial surgery navigation aided by a compact intraoperative magnetic resonance imager. J Neurosurg 2001;94:936-45

6. Sutherland GR, Kaibara T, Louw D, et al. A mobile high-field magnetic resonance system for neurosurgery. J Neurosurg 1999;91:804-13
7. Yrjana SK, Katisko JP, Ojala RO, et al. Versatile intraoperative MRI in neurosurgery and radiology. Acta Neurochir (Wien) 2002;144:271-78

8. Schwartz RB, Hsu L, Wong TZ, et al. Intraoperative MR imaging guidance for intracranial neurosurgery: experience with the first 200 cases. Radiology 1999;211:477-88

9. Martin AJ, Hall WA, Liu H, et al. Brain tumor resection: intraoperative monitoring with high-field-strength MR imaging-initial results. Radiology 2000;215:221-28

10. Nimsky C, Ganslandt O, Von KB, et al. Intraoperative high-field-strength MR imaging: implementation and experience in 200 patients. Radiology 2004;233:67-78

11. Black PM, Moriarty T, Alexander E III, et al. Development and implementation of intraoperative magnetic resonance imaging and its neurosurgical applications. Neurosurgery 1997;41:831-42

12. Truwit $\mathrm{CL}, \mathrm{Hall} \mathrm{WA}$. Intraoperative magnetic resonance imaging-guided neurosurgery at 3-T. Neurosurgery 2006;58:ONS-338-45

13. McGee KP, Lee KH, Ward H, et al. Development of a two-room MR/OR suite concept to clinical implementation. Presented at the Joint Annual Meeting of ISMRM-ESMRMB, May 19-25, 2007; Berlin, Germany. Abstract No 1111.

14. Knauth M, Aras N, Wirtz CR, et al. Surgically induced intracranial contrast enhancement: potential source of diagnostic error in intraoperative MR imaging. AJNR Am J Neuroradiol 1999;20:1547-53 